\title{
Considerations on prostate cancer: diagnosis and treatment decisions
}

\author{
Thomas R. W. Herrmann • Axel S. Merseburger • \\ Martin Burchardt
}

Received: 28 August 2009 / Accepted: 2 September 2009 / Published online: 15 September 2009

(C) Springer-Verlag 2009

Discussion on the accuracy of PSA and biopsy for the selection of tailored treatment has been generated from several fields. The inherent variability of total, free and complex PSA has been demonstrated. Analytical sample handling, laboratory processing, assay performance, and standardisation as well as biological variation are factors influencing its impact. Bögermann and colleagues from the University of Essen, Germany draw attention to the fact that different pre-analytic timetables of blood samples in inpatient and out-patient setups lead to subsequent different fPSA degradation, thereby shedding light on the need for different cut-off values for biopsy indication in these clinical settings.

Prostate biopsy has evolved from a paradigm of a small number of random biopsies to a systematic, numerically standardised, and anatomically controlled strategy. Over the past several years, data have demonstrated the need for defined biopsy strategies for detecting, accurately grading, and staging prostate cancer in varying patient populations. Saturation biopsy schemes have proven beneficial in repeat biopsy and active surveillance protocols. Ploussard and colleagues from Creteil, Paris, France, report on how to follow-up prostate cancer detection with repeated biopsies after high-grade prostatic intraepithelial neoplasia (HGPIN) and atypical small acinar proliferation (ASAP) following their initial 21-core extended biopsy scheme.

Proving the sensitivity and specificity of artificial neuronl networks (ANN) for prostate cancer in a different "statistical environment" is a necessary part of the evaluation

T. R. W. Herrmann $(\bowtie) \cdot$ A. S. Merseburger · M. Burchardt Department of Urology and Urologic Oncology, Hannover Medical School, Carl-Neuberg-Straße 1, 30625 Hannover, Germany

e-mail: Herrmann.Thomas@mh-hannover.de of any ANN. Meijer and colleagues from Hertogenbosch, The Netherlands, report on their results on prostate biopsy predicting model of the ANN Prostataclass of the Universitätsklinikum Charité, Berlin, Germany, from their database.

Trifecta (continence, potency and oncological result) has been the issue of discussions in recent years but lacking single center data for intra-institutional comparisons. Drouin and colleagues from Pitié-Salpétrière, University of Paris, France, compared the oncological midterm outcome over 50 months of patients treated during the same period in a single institution. Gilberti and colleagues from Savona, Italy present a prospective randomised trial including the mid-term follow-up data comparing brachytherapy with radical prostatectomy with regard to Trifecta.

As with any other surgical treatment option, laparoscopy carries the risk of major surgical complications. Teber and colleagues from Heilbronn, Germany, present their experience with identifying and treating ureteral injury during laparoscopic radical prostatectomy in their institutions series of more than 2,100 patients.

Rinnab and colleagues from the University of Ulm, Germany have been involved in detection methods for patients with biochemical recurrence after treatment of prostate cancer, which remains a diagnostic and therapeutic challenge. Here, they display their results on [(11)C] choline PET/CT in patients with biochemical recurrence after radical prostatectomy.

Androgen-deprivation therapy is associated with important adverse effects which have an impact on quality of life, especially in men over 70 years of age. A further disadvantages of continuous application is the cost consideration. Numerous phase II trials have shown the efficacy of intermittent androgen withdrawal with a reduction in inter-treatment intervals. Prapotnich and colleagues from Montsouris, Paris, France, report their oncological results over a period 
of 16 years with intermittent androgen deprivation therapy in advanced prostate cancer.

The high demand for definitive treatment of prostate cancer in older patient cohorts is a challenge for physicians. Many patients over 70 years of age regularly receive surgical treatment. Pfitzenmayer and colleagues from the University of Heidelberg, Germany, report on survival of their patients in this category after radical prostatectomy in comparison to younger patients cohorts.

Treatment patterns, especially for high-risk or locally advanced cancer, have become a much-discussed topic lately. Jeong and colleagues from Seoul National University, Korea, focus on the effect of concomitant pulmonary obstructed disease and its outcome after surgical treatment. Another article from Pitié-Salpétrière, University of Paris, France presented by Nyguen and colleagues focuses on the outcome of high-risk patients with a PSA level above $20 \mathrm{ng} / \mathrm{ml}$.

This issue presents a fascinating overview on diverse mainstream topics in prostate cancer and provides the reader with the most up-to-date scientific results in basic and clinical research. 